\title{
Field Weed Recognition Based on an Improved VGG With Inception Module
}

\author{
Lifang Fu, Northeast Agricultural University, China \\ Xingchen Lv, Northeast Agricultural University, China \\ Qiufeng Wu, Northeast Agricultural University, China \\ (iD) https://orcid.org/0000-0002-4787-2549 \\ Chengyan Pei, Northeast Agricultural University, China
}

\begin{abstract}
The precision spraying of herbicides can significantly reduce herbicide use, and recognizing different field weeds is an important part of it. In order to enhance the efficiency and accuracy of field weed recognition, this article proposed a field weed recognition algorithm based on VGG model called VGG Inception (VGGI). In this article, three optimizations were made. First, the reduced number of convolution layers to reduce parameters of network. Then, the Inception structure was added, which can maintain the main features, and have better classification accuracy. Finally, data augmentation and transfer learning methods were used to prevent the problem of over-fitting, and further enhance the field weed recognition effect. The Kaggle Images dataset was used in the experiment. This work achieved greater than $98 \%$ precision in the detection of field weeds. In actual field, the accuracy could reach $80 \%$. It indicated that the VGGI model has an outstanding identification performance for seedling, and has significant potential for actual field weed recognition.
\end{abstract}

\section{KEYWORDS}

Data Augmentation, Inception Structure, Transfer Learning, VGG Model, Weed Recognition

\section{INTRODUCTION}

Recognizing and removing weed is an important part of field management. Weeds secretions hinder the growth of seedlings and cause the yield reduction in the field (Su, 2017). In addition, weeds are main medium to spread diseases and insect pests (Zhao \& Liu, 2019). Weeds compete for environmental resources such as nutrients, sunlight, and water (Hu, 2007). Manual weeding (Xie et al., 2018) and biological weed control (Shabbir et al., 2018) are primitive and traditional methods for weeds control in China (Lv, Dong, Sun, \& Li, 2018). On the one hand, the cost for the research work and development of biological weed control is very high (Chen \& Qiang, 2015), on the other hand, chemical weeding (Wen, Ying, \& Libai, 2007) pollutes the environment (Liu, Wang, \& Guan, 2005). There is an urgent need to improve the efficiency of weeds recognition and removing. 
The versatility of computer vision has made it a fit tool used in many fields, including precision agriculture (Andrea, Daniel, \& Misael, 2017). Machine vision technology was first incorporated into crop detection algorithms to identify crop rows and to segment plants and weeds (Brivot \& Marchant, 1996). Researchers developed an automated crop spraying system, and analysised the green histogram of plants images and performed crop and weed segmentation with unspecified classifiers (Aitkenhead et al., 2003). Cao, Wang, Mao, and Zhang (2007) developed a method to identify weeds in field by using position and texture features of drill crops, the method could achieve $93 \%$ accuracy. Qiao, He, Zhao, and Tang (2013) developed and tested a method of weed recognition based on multi-spectral images and SVM classifier, this method spent less time than traditional SVM classifier. However, this method had a limitation that the accuracy needs to be improved. Wang and $\mathrm{Li}$ (2016) proposed an algorithm to extract the height feature of the image and then fuse it with the texture to identify and obtain a higher recognition rate, the algorithm had an effectiveness of $98.33 \%$. However, the plant detection algorithm presented a limitation that the method pent extremely large computing time. Yan (2018) proposed a method to identify weeds by removing shape and color of images, the goal of which was to prepare for intelligent inter-tillage in fields. In order to improve accuracy of crop and weed identification, a fast field weed identification method was proposed based on the deep convolution network and binary code, the method could achieve 92.7\% accuracy (Jiang et al., 2018). However, the effectiveness of the approach was not guaranteed in some production crop scenarios.

In recent years, with the development of computer technology, the application of machine vision recognition method in the identification of weeds and crops is also very extensive. Machine vision technology can provide important tools for real-time image processing and weed detection. The convolution neural network (CNN) (Lecun and Bengio, 1998) have been widely used in various fields and also have achieved remarkable results in the field of image recognition. One of the major advantages is that the original image can be used as an input and the automatic training feature, and further reduce the manual pre-processing. Especially, LeNet, VGG16 (Ke et al., 2018; Zhang et al., 2018; Zhuo et al., 2018), GoogLeNet (Szegedy et al., 2014; Zhai et al., 2019; Xie et al., 2018), ResNet (Gu et al., 2019) and other classical neural network, they all have achieved excellent results in image classification and recognition. Here, an VGG Inception (VGGI) model was proposed to identify and detect field seedlings and weeds. In this paper, three optimizations were made. First, the reduced number of convolution layers to reduce parameters of network. Then, the Inception module was added and dropout operations, which can maintain the main features, have better classification accuracy and are more robust. Finally, data augmentation and transfer learning methods were used to prevent the problem of over-fitting, and further enhance the field weed recognition effect. Moreover, the VGGI model applied to the actual images, the algorithm preformed a good effectiveness.

\section{MATERIALS AND METHODS}

In this section, we describe the characteristics of the dataset in detail, introduce the model in this paper and related model.

\subsection{Dataset}

In this paper, the weeds and crops image datasets of Kaggle competition were used to train and test the network model. According to Xu (2011), the dataset includes six kinds of weeds, Black-grass, Charlock, Cleavers, Common Chickweed, Fat Hen, Loose Silky-bent and six kinds of crops, Common wheat, Maize, Scentless Mayweed, Shepherds Purse, Small-flowered Cranesbill, Sugar beet. The total of 4750 field weed samples of 12 categories were depicted in Table 1.

All the images are patched of $224 \times 224$ pixels by Matlab. The number of images were randomly divided into two groups: $80 \%$ of images as the training data and $20 \%$ of images as testing data. As shown in Figure 1, which is the example of field plants. 
Table 1. Details of the field weeds and crops image dataset

\begin{tabular}{|c|c|c|c|}
\hline Labels & Category & Numbers & Image Features \\
\hline 1 & Black grass & 263 & The leaves are slender and the edges are finely jagged. \\
\hline 2 & Charlock & 390 & $\begin{array}{l}\text { The leaves are elliptical, apex or sharp, base round or wedge- } \\
\text { shaped, with scalloped edges. }\end{array}$ \\
\hline 3 & Cleavers & 287 & $\begin{array}{l}\text { The leaves are paper, generally } 5-8 \text { pieces, with needle-like } \\
\text { convex tips at the top, and the base is tapered. }\end{array}$ \\
\hline 4 & Common Chickweed & 611 & $\begin{array}{l}\text { The leaves are ovate, apex acuminate, base attenuate or nearly } \\
\text { cordate, margin entire. }\end{array}$ \\
\hline 5 & Common wheat & 221 & Incomplete period, blade slender. \\
\hline 6 & Fat Hen & 475 & $\begin{array}{l}\text { The leaves are rhomboid-ovate to lanceolate, apex slightly } \\
\text { pubescent, base cuneate to broadly cuneate. }\end{array}$ \\
\hline 7 & Loose Silky bent & 654 & $\begin{array}{l}\text { The leaf sheath is closed and the apex of the leaf tongue is } \\
\text { nearly circular. }\end{array}$ \\
\hline 8 & Maize & 221 & $\begin{array}{l}\text { The leaves are flat and wide at the seedling stage, and the base } \\
\text { is round and ear-shaped. }\end{array}$ \\
\hline 9 & Scentless Mayweed & 516 & The leaves are slender, apex blunt, base cuneate. \\
\hline 10 & Shepherds Purse & 231 & $\begin{array}{l}\text { The leaves are oblong, the top is linear, the base is ear-shaped, } \\
\text { and the edges are serrated. }\end{array}$ \\
\hline 11 & $\begin{array}{l}\text { Small-flowered } \\
\text { Cranesbill }\end{array}$ & 496 & Stems erect, leaves round, with rounded teeth. \\
\hline 12 & Sugar beet & 385 & $\begin{array}{l}\text { Stems erect, leaves round, with long petiolate, apex blunt, } \\
\text { base cuneate. }\end{array}$ \\
\hline & Total & 4750 & \\
\hline
\end{tabular}

\subsection{VGG Model}

VGG is the champion of the 2014 ImageNet competition (ILSVRC14, 2014), the model is developed by AlexNet. The VGG model has two characteristics: The first is that the convolution kernels are small, most of size are $3 \times 3$, and few are $1 \times 1$. The convolution operation is accompanied by an activation function, which can identify more abundant features; the second is the small pooling kernel, compared to AlexNet's $3 \times 3$ pooling kernel, VGG only has pooling kernel of size $2 \times 2$, making the layers deeper, the feature map can be wider.

VGG16 is one of the most classic neural networks, as shown in Figure 2, conv1 and conv2, each contains two convolutional layers, conv3, conv4 and conv5, each contains 3 convolutional layers, $\mathrm{fc} 1, \mathrm{fc} 2$ and $\mathrm{fc} 3$, each contains a fully-connected layer; the last one is the image feature classification layer, they have a total of 16 layers. Although VGG16' effect is better, convergence is faster, but it also has some disadvantages. With the deepening of model level, the training parameters is becoming more complex, it will take a lot of time, what we do is to solve these problems.

\subsection{Inception Module of GoogLeNet}

The GoogLeNet model is a deep learning structure, proposed by Christian Szegedy and won the championship in the classification and testing of ILSVRC-2014, where it achieved 5.5\% top-5 classification error on the ImageNet challenge, compared to AlexNet's $15.3 \%$ top-5 classification error. As Zhong et al. (2015) noted that GoogLeNet model has improved AlexNet's problems of overfitting, complexity computation and low accuracy, also has high robustness. More importantly, it also introduced a new module called "Inception". GoogLeNet is an inception architecture that combines 
Figure 1. Sample of field weeds and crops train dataset (Note: From left to right and from top to bottom, there are black-grass, charlock, cleavers, common chickweed, common wheat, fat hen, loose silky-bent, maize, scentless mayweed, shepherd purse, small-flowered cranesbill and sugar beet, respectively)
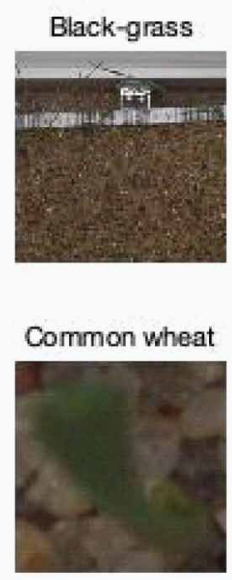

Scentless Mayweed

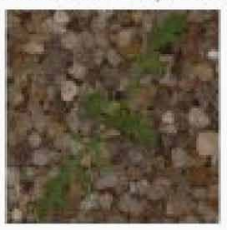

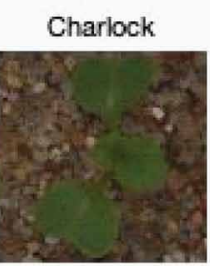

Fat Hen
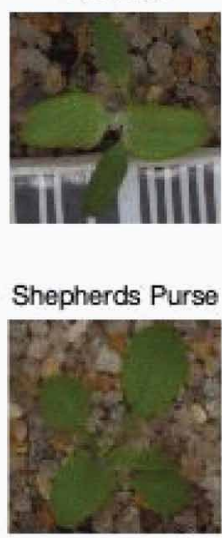
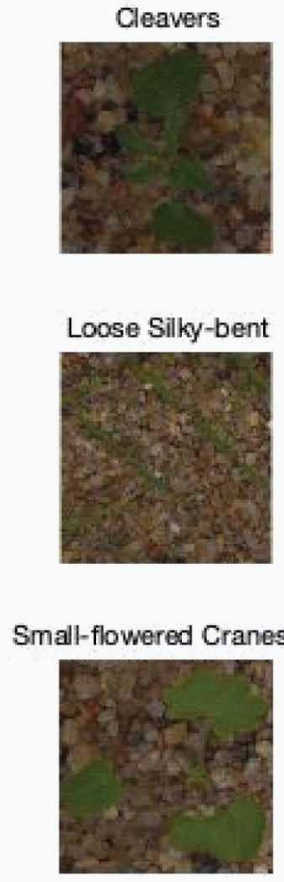

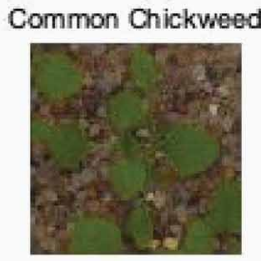

Maize

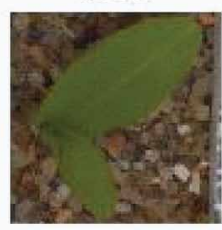

Sugar beet

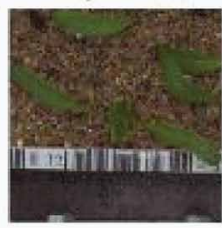

Figure 2. VGG16 Model

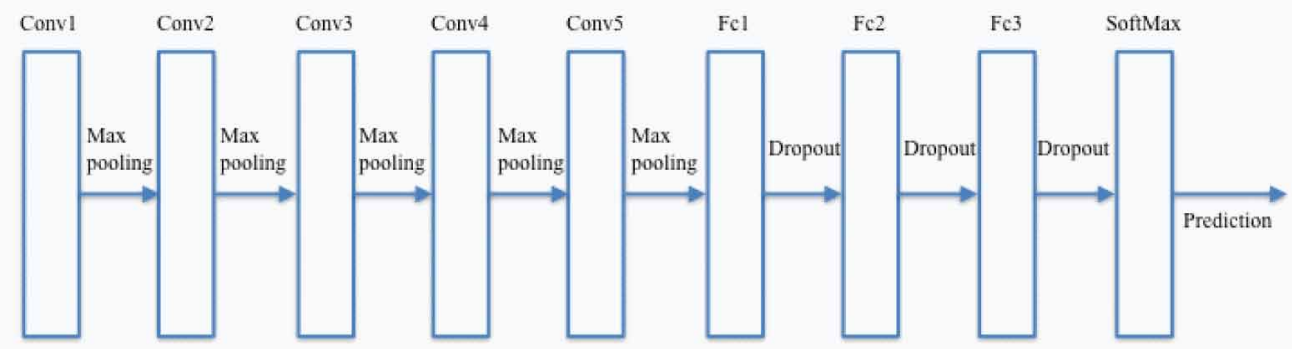

the multi-scale idea and dimension reduction layers based on the Hebbian principle and embedding learning, as shown in Figure 3. Huang (2017) pointed that this structure greatly increases the depth and width of the network, seeks the optimal spatial structure, and repeats in space to obtain more image features, while keeping the calculation constant. Overall, Inception module plays a vital role and promotes the development process of CNN.

\subsection{VGGI Model Structure}

In our experiment, we improved VGG model by reducing layers of convolution and increasing Inception modules. The new model contains Inception module and dropout operations. Inception module not only can increase the depth and width of network, but also can reduce parameters of network greatly. Then average pooling and dropout added. Average pooling can divide the images into several blocks 
Figure 3. Inception modul of the model (Note: The Inception module is composed of 7 convolution layers, including four $1 \times$ 1 convolutional layer, one $3 \times 3$ convolutional layer and one $5 \times 5$ convolutional layer, and one $3 \times 3$ maximum pooling layer)

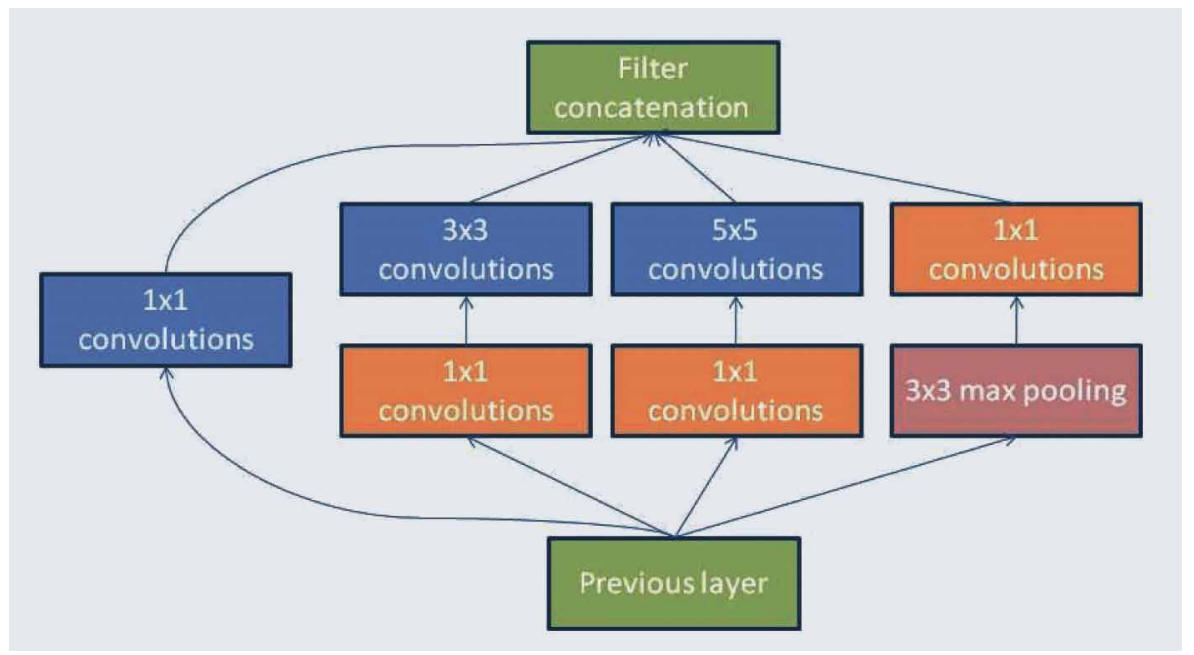

of the same size, and only takes the largest value in each block. After abandoning other nodes, it maintains the original plane structure invariance, and further reduces the amount of computation by dropout. The improved model is shown in Figure 4, and the layer information as follows:

- Input layer: Loading the images, after preprocessing, the 3-channel field weed images are directly as input to the convolutional layer;

- Convolutional layer: A convolutional layer is characterized by sparse local connectivity and weight sharing. Each neuron of the layer is only connected to a small local area of the input. Different neurons respond to different local areas of the input, which overlap with each other to obtain a better representation of the image. In addition, the neurons of a convolutional layer are grouped in feature maps sharing the same weights, so the entire procedure becomes

Figure 4. Structure of the VGGI model (Note: In the VGGI model, the first nine convolutional layers are all used $3 \times 3$ convolution kernels stacking. The two $3 \times 3$ convolution kernel stacks are equivalent to a $5 \times 5$ convolution kernel and three $3 \times 3$ convolution kernels are equivalent to a $7 \times 7$ convolution kernel. After the stacking of each convolution kernel, four Inception modules are followed, the maximum pooling layer is added in the middle of two Inceptions, and the final Dropout layer is used to reduce the parameters. The fully connected layer is used to output at the end of the network. SoftMax is used to calculate the loss.)

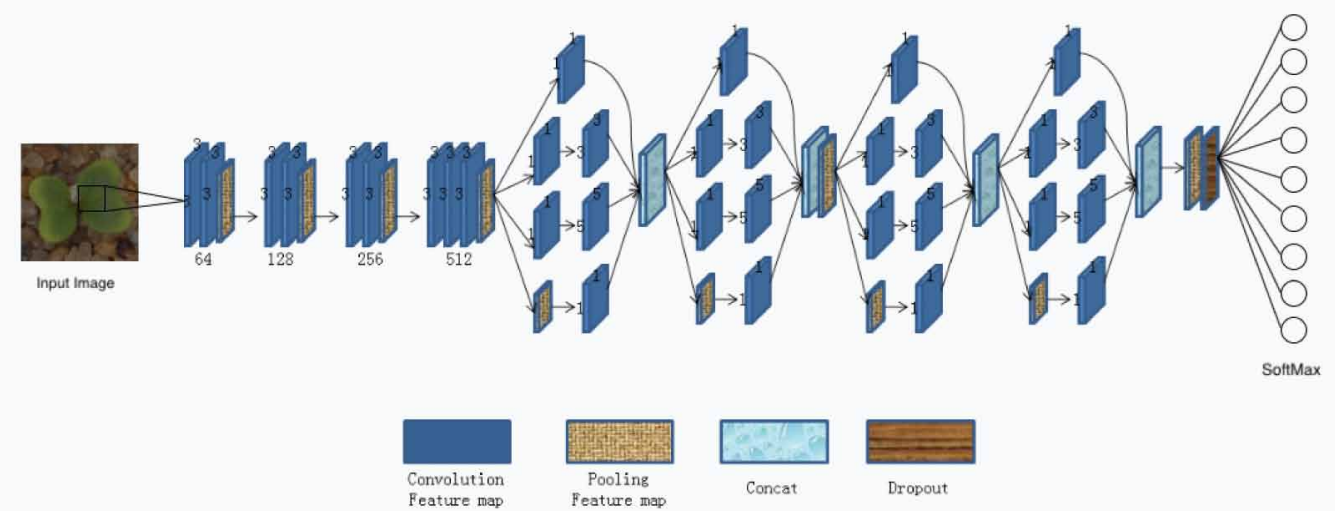


equivalent to convolution, with the shared weights being the filters for each map. Weight sharing drastically reduces the number of parameters of the network and hence increases efficiency and prevents overfitting. This model has a total of 9 convolution kernels of size $3 \times 3$, the first two convolutional layers contains 64 feature maps, it followed by a pooling operation. The pooling kernel of size $2 \times 2$ with a stride of 2 pixels, this way is max-pooling. Then 2 convolutional layers contain the feature map size of 128 , followed by the pooling kernel size of $2 \times 2$ with a stride of 2 pixels. Next 2 convolutional layers contain the feature map size of 256 , followed by the pooling kernel size of $2 \times 2$ with a stride of 2 pixels. The last 3 convolutional layers contains feature maps size of 512, followed by the pooling kernel size of $2 \times 2$ with a stride of 2 pixels;

- Activation function: Each convolution layer is followed by the Rectifier Linear Unit (ReLU) (Vinod, Geoffrey, and Hinton, 2010) activation function, which is an unsaturated function. The sparsity of the calculated results can reduce the backpropagation error, accelerate the convergence speed of the network, improve the nonlinear expression ability and improve the identification ability;

- Inception module: Four Inception modules are followed, the maximum pooling layer is added in the middle of two Inceptions, and the final dropout layer is 0.4 , which used to reduce the parameters. The fully connected layer is used to output at the end of the network. SoftMax is used to calculate the loss.

This paper discussed the influences of various parameters in the model on the accuracy. Then, compared and analyzed the evaluation indicators (precision, recall, and F1 scores) of the traditional VGG model and VGGI by confusion matrix.

\section{EXPERIMENTAL RESULTS AND ANALYSIS}

In this section, we discuss the experimental results. All experiments were implemented in Matlab under Windows10, using the GPU NVIDIA GTX1050. In this work, overall accuracy, precision, recall and F1 score were selected as the quantitative evaluation index.

\subsection{Data Augmentation}

Due to the lack of a large number of filed weeds data, especially the black-grass, charlock, cleavers, common wheat, maize and shepherd purse, it is obvious that the training of the model easily leads to over-fitting problem and inaccurate accuracy. Therefore, based on the original field weeds dataset, we adopt a combination of two solutions.

One is the data augmentation. We use reflection deformation, the image data for 30 degrees, 60 degrees and vertical mirror of rotation. In addition, to simulate the real field environment, the filed weed images corrupted with $20 \%$ salt and pepper noise, and used the adaptive median filter to enhance the images. Expanding from over 4,000 in 12 categories of signs to 20,944. The number of final filed weed data are shown in Table 2. The augmentation samples are shown in Figure 5.

Another one is the transfer learning method, we used parameters of VGG16 as the parameters of stacking convolution layer of VGGI, and parameters of GoogLeNet's Inception module as parameters of VGGI's Inception module. Then fine-tuning training for the last three layers of VGGI. Fine-tune training can keep the basic characteristics of dataset, such as color, texture, etc. At the same time, fine-tune training can avoid overfitting. It is very useful for many tasks.

In our work, we use data augmentation and transfer learning method of further improve the VGGI model. Data augmentation mainly affects the input stage of the model and enhances the quantity and uniqueness of data. Transfer learning mainly plays an important role in model parameter. 
Table 2. The final filed weeds data

\begin{tabular}{|l|l|l|l|l|l|l|}
\hline \multicolumn{1}{|c|}{ Category } & Black-Grass & Charlock & \multicolumn{1}{|c|}{ Cleavers } & \multicolumn{1}{c|}{$\begin{array}{c}\text { Common } \\
\text { Chickweed }\end{array}$} & $\begin{array}{c}\text { Common } \\
\text { Wheat }\end{array}$ & Fat Hen \\
\hline Training set & 1219 & 1463 & 1590 & 1647 & 1216 & 1346 \\
\hline Testing set & 304 & 366 & 397 & 412 & 304 & 336 \\
\hline Category & $\begin{array}{l}\text { Loose Silky } \\
\text { bent }\end{array}$ & Maize & $\begin{array}{l}\text { Scentless } \\
\text { Mayweed }\end{array}$ & $\begin{array}{l}\text { Shepherds } \\
\text { Purse }\end{array}$ & $\begin{array}{l}\text { Small- } \\
\text { flowered } \\
\text { Cranesbill }\end{array}$ & Sugar beet \\
\hline Training set & 1218 & 1310 & 1512 & 1386 & 1458 & 1392 \\
\hline Testing set & 304 & 328 & 378 & 346 & 364 & 348 \\
\hline
\end{tabular}

Figure 5. Samples of the weed image data augmentation
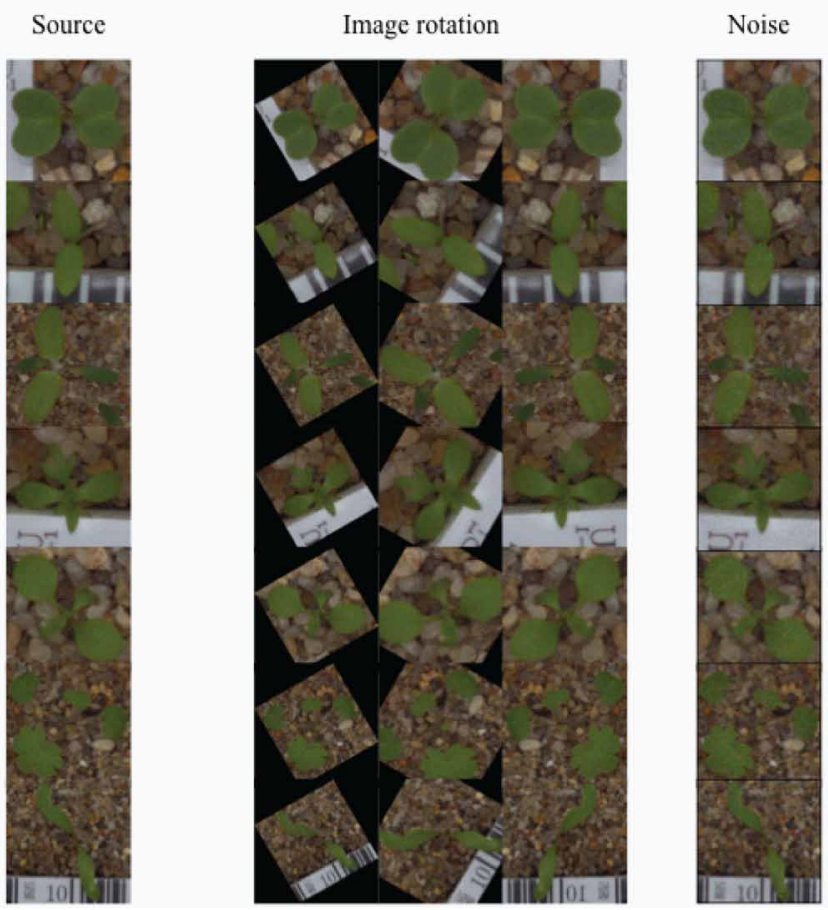

\subsection{Model Parameters}

The classification accuracy of VGG and VGGI in various batch size and epochs was shown in Table 2. In Table 3, the column1 and column 4 represents different batch size and epochs on VGG and VGGI model. Give an example, VGG $(16,30)$ represents that the pre-trained network used is VGG, and the batch size of the network was set to 16 , the number of epochs was set to 30 . Some hyperparameters were set as follows: the optimization method was stochastic gradient descent (SGD) with momentum, and the momentum was set to 0.9 , and the initial learning rate was set to 0.001 .

By comparing the classification accuracy of the VGG and VGGI model, it can be found that the performance of $\operatorname{VGGI}(64,30)$ model and $\operatorname{VGG}(32,30)$ are relatively optimal. Therefore, $\operatorname{VGGI}(64,30)$ and $\operatorname{VGG}(32,30)$ were used to explore the impact of its network structure on classification performance subsequently. 
Table 3. Test accuracy of different models

\begin{tabular}{|l|l|l|l|l|l|}
\hline \multicolumn{1}{|c|}{ Model } & \multicolumn{1}{c|}{ Accuracy $(\%)$} & \multicolumn{1}{c|}{ Time(s) } & \multicolumn{1}{c|}{ Model } & \multicolumn{1}{c|}{ Accuracy (\%) } & \multicolumn{1}{c|}{ Time(s) } \\
\hline VGG(32,10) & 95.63 & 39.89 & VGGI(32,10) & 95.99 & 40.47 \\
\hline VGG(64,10) & 97.09 & 40.53 & VGGI(64,10) & 94.05 & 37.68 \\
\hline VGG(16,20) & 98.19 & 40.77 & VGGI(16,20) & 95.73 & 52.66 \\
\hline VGG(32,20) & 98.33 & 45.05 & VGGI(32,20) & 97.64 & 43.42 \\
\hline VGG(64,20) & 98.14 & 52.59 & VGGI(64,20) & 98.45 & 47.40 \\
\hline VGG(32,30) & $\mathbf{9 8 . 4 9}$ & $\mathbf{4 0 . 3 6}$ & VGGI(32,30) & 98.04 & 51.54 \\
\hline VGG(64,30) & 97.89 & 40.35 & VGGI(64,30) & $\mathbf{9 8 . 7 3}$ & $\mathbf{3 8 . 5 8}$ \\
\hline VGG(32,40) & 96.44 & 46.67 & VGGI(32,40) & 96.97 & 46.58 \\
\hline VGG(64,40) & 95.98 & 47.53 & VGGI(64,40) & 95.75 & 49.67 \\
\hline
\end{tabular}

\subsection{Analysis of Experimental Results}

In order to quantitatively analyze and test the network performance, the precision $(\mathrm{P})$, recall $(\mathrm{R})$ and $\mathrm{F} 1$ scores $(\mathrm{F} 1)$ were used for objective evaluation. The precision is the percentage of the correct portion of the test results. The recall is the percentage of the correct part of the test results to the actual correct part. In addition, the F1 score was given to evaluate the overall performance of the classifier. The calculation formulas are:

$$
\begin{aligned}
& \text { precision }=\frac{\text { true positive }}{\text { true positive }+ \text { false positive }} \\
& \text { recall }=\frac{\text { true positive }}{\text { truepositive }+ \text { falsenegative }} \\
& F 1=\frac{2 \times \text { precision } \times \text { recall }}{\text { precision }+ \text { recall }}
\end{aligned}
$$

where, true positive refers to the number of correctly identified crops, false positive refers to the number of incorrectly identified crops, false negative refers to the number of incorrectly identified crops as weeds.

The total 4188 field weed samples of 12 categories of testing set were tested, and the results were shown in Table 4.

As shown in Table 4, the average precision of VGG model is $90.79 \%$, the average precision of the VGGI model is $98.63 \%$, the average recall of VGG is $98.31 \%$, the average recall of the VGGI model is $98.64 \%$, the average F1 score of VGG is $98.30 \%$, and the average F1 score of the VGGI model is $98.63 \%$.

Kamilaris and Prenafeta-Boldu (2018) pointed out that in order to avoid the removal of crops as weeds, it is allowed to have small precision but large recalls. The best case is high precision, high recall and high F1 score. Overall, the VGGI model performs better than the VGG model in the test of field weed images.

Sampling of misclassified by the VGGI model in the testing set, these typical misclassified samples are shown in Figure 6. According to the confusion matrix in Table 4, the main causes of misclassification are summarized into the following categories: 
Table 4. Confusion Matrix and classification performance of the model

\begin{tabular}{|c|c|c|c|c|}
\hline Method & Labels & Precision (\%) & Recall (\%) & F1(\%) \\
\hline \multirow{12}{*}{ VGGI } & Black grass & 95.03 & 94.10 & 94.56 \\
\hline & Charlock & 100.00 & 98.91 & 99.45 \\
\hline & Cleavers & 99.50 & 99.50 & 99.50 \\
\hline & Common Chickweed & 99.51 & 99.51 & 99.51 \\
\hline & Common wheat & 99.34 & 99.34 & 99.34 \\
\hline & Fat Hen & 100.00 & 100.00 & 100.00 \\
\hline & Loose Silky bent & 94.75 & 95.07 & 94.91 \\
\hline & Maize & 97.62 & 100.00 & 98.80 \\
\hline & Scentless Mayweed & 99.21 & 99.47 & 99.34 \\
\hline & Shepherds Purse & 98.85 & 99.13 & 98.99 \\
\hline & Small-flowered Cranesbill & 100.00 & 99.45 & 99.72 \\
\hline & Sugar beet & 99.71 & 99.14 & 99.42 \\
\hline \multirow{12}{*}{ VGG } & Black grass & 90.16 & 96.27 & 93.11 \\
\hline & Charlock & 99.19 & 100.00 & 99.59 \\
\hline & Cleavers & 100.00 & 98.74 & 99.37 \\
\hline & Common Chickweed & 99.27 & 99.27 & 99.27 \\
\hline & Common wheat & 97.73 & 99.34 & 98.53 \\
\hline & Fat Hen & 99.40 & 98.51 & 98.95 \\
\hline & Loose Silky bent & 96.27 & 89.27 & 92.64 \\
\hline & Maize & 100.00 & 99.70 & 99.85 \\
\hline & Scentless Mayweed & 100.00 & 99.47 & 99.73 \\
\hline & Shepherds Purse & 98.58 & 100.00 & 99.28 \\
\hline & Small-flowered Cranesbill & 100.00 & 99.73 & 99.86 \\
\hline & Sugar beet & 99.43 & 99.43 & 99.43 \\
\hline
\end{tabular}

1. The main reason of confusion between black-grass and loose silky-bent is that the size and shape of them are similar, the figure of the back is the same, and the proportion of plants is small, so it is easy to be confused in identification and classification. Similarly, common wheat is also easy to be mistakenly divided into black grass;

2. Both the cleavers and common chickweed have two morphological features, both of them have nearly elliptical shape and similar edges, so they were misclassified.

\subsection{Prediction of Actual Effect}

In order to verify the actual field prediction effect of the optimal model in this paper, we photographed 2 common chickweeds, 3 maizes and 1 common wheats for testing from field. The sample of all the testing data is shown in Figure 7. The optimal parameter VGGI model was used to make the prediction. 3 options with the highest prediction probability are output, which are arranged in descending order from top to bottom. From the Figure 8, the actual field prediction effect of maize can reach the accuracy of $80 \%$, and the actual field prediction effect of common wheat can reach the accuracy of $72 \%$. But 
Figure 6. Samples of misclassified

Image to Recognize

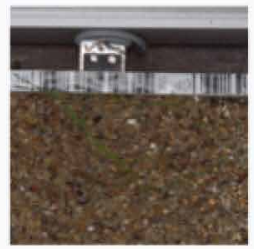

Forecast: Loose Silky-bent

Fact: Black-grass

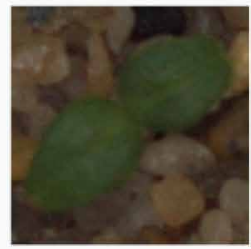

Forecast: Common Chickweed

Fact: Cleavers

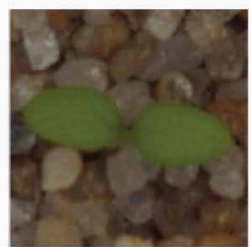

Image to Recognize

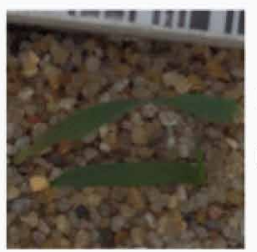

Forecast: Black-grass

Fact: Common wheat

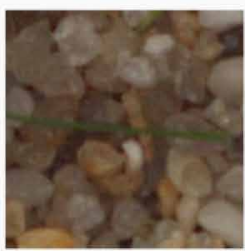

Forecast: Black-grass

Fact: Loose Silky-bent

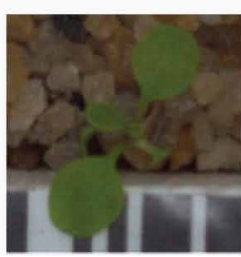

Forecast: Scentless Mayweed

Fact: Shepherds Purse

Figure 7. Sample of field weeds and crops testing data. (No.0 No.2 are Maize, No.3 No.4 are Common Chickweed, No.5 is Common wheat.)

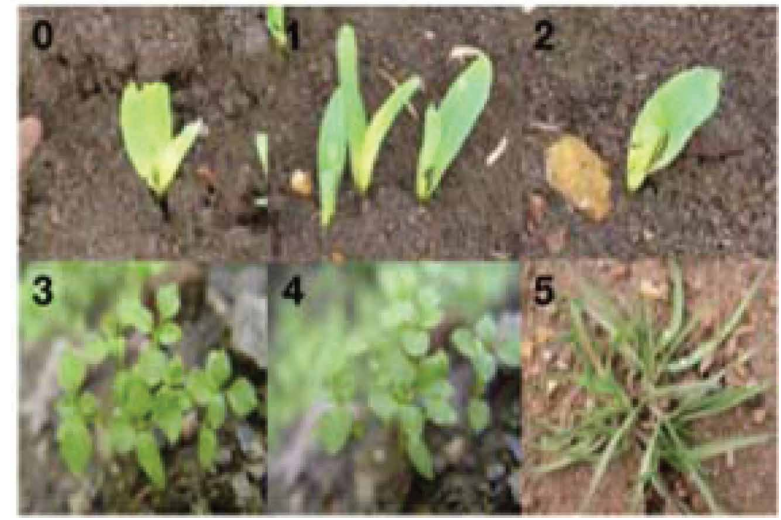

the actual field prediction effect of common chickweed is not good, only the accuracy of $30 \%$. It may be because the common chickweed background is too complex and the shooting angle is different.

\section{CONCLUSION}

In this work, improved VGG with Inception module (VGGI) for field weed recognition was proposed, in which the multi-layer continuous convolutions were reduced and the Inception modules were added. By using data augmentation and transfer learning methods, both the network training efficiency and the convergence rate were enhanced. It is proved that the VGGI model can learn more information and 
Figure 8. Actual Field Effect Prediction Diagram of VGGI. (The images in first column are respectively No.0, No.1, No.2. The images in second column are respectively No.3, No.4, No.5.)
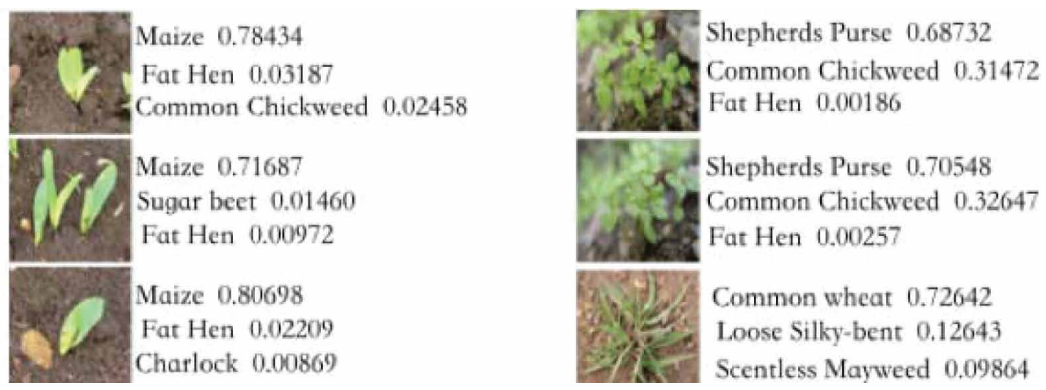

extract more features effectively, these advantages ensure the network's convergence and robustness. The hyper-parameters of the convolutional neural network were set by a lot of experiments. As it shown in the result, the classification accuracy of the model got to as high as $98.73 \%$, while the computing time was only 38.58 seconds. This indicates that the new model can accurately identify field crops, weeds with large data, prepared for accurate weed removal in the future, and provided reliable support.

However, the VGGI model is still deficient in recognizing the complex background and different shooting angles images. We will focus on how to enhance the identification of field weeds under the complex background and different shooting angles in the future work.

Funding

Chinese Postdoctoral Science Foundation (2014M561321)

National Science and Technology Support Plan (2018YFD0300105-2) 


\section{REFERENCES}

Aitkenhead, M. J., Dalgetty, I. A., Mullins, C. E., McDonald, A. J. S., \& Strachan, N. J. C. (2003). Weed and crop discrimination using image analysis and artificial intelligence methods. Computers and Electronics in Agriculture, 39(3), 157-171. doi:10.1016/S0168-1699(03)00076-0

Andrea, C. C., Daniel, B. B. M., \& Misael, J. B. J. (2017). Precise weed and maize classification through convolutional neuronal networks. In Paper presented at the IEEE Ecuador Technical Chapters Meeting (ETCM). IEEE press. doi:10.1109/ETCM.2017.8247469

Brivot, R., \& Marchant, J. (1996). Segmentation of plants and weeds for a precision crop protection robot using infrared images. IEE Proceedings. Vision Image and Signal Processing, 143(2), 118-124. doi:10.1049/ ip-vis: 19960202

Cao, J. J., Wang, Y. M., Mao, W. H., \& Zhang, X. C. (2007). A method of weed identification in wheat field based on texture and location. Agricultural Machinery, 38(4), 107-110.

Chen, S. G., \& Qiang, S. (2015). The Status and Future Directions of Bioherbicide Study and Development. Zhongguo Shengwu Fangzhi Xuebao, 31(5), 770-779.

Gu, W. H., Zhu, W., Chen, X. D., Zheng, B. B., \& He, L. F. (2019). Research on Fatigue Detection Method Based on Multiscale Pooled Convolutional Neural Network. Jisuanji Yingyong, (11): 1-9.

Hu, R. (2007). Research on Identification of Weeds in Maize Seedling Based on Digital Image Processing. Wuhan: Huazhong Agricultural University.

Huang, S. P., Sun, C., Ma, Xu., \& Qi, L. (2017). Detection method of rice panicle disease based on deep convolutional neural network. Nongye Gongcheng Xuebao (Beijing), 33(20), 169-176.

ImageNet Large-Scale Visual Recognition Challenge. (2014). Retrieved from http://image-net.org/challenges/ LSVRC/2014/index

Jiang, H. H., Wang, P. F., Zhang, Z., Mao, W. H., Zhao, B., \& Qi, P. (2018). Rapid identification of weeds in corn fields based on convolutional networks and hash codes. Nongye Jixie Хиеbao, 49(11), 37-45.

Kamilaris, A., \& Prenafeta-Boldu, F. X. (2018). Deep learning in agriculture: A survey. Computers and Electronics in Agriculture, 17(1), 70-90. doi:10.1016/j.compag.2018.02.016

Ke, H., Chen, D., Li, X., Tang, Y. B., Shah, T., \& Ranjan, R. (2018). Towards brain big data classification: Epileptic EEG identification with a lightweight VGGNet on Global MIC. IEEE Access, 2(99), 14722-14733. doi:10.1109/ACCESS.2018.2810882

Lecun, Y., \& Bengio, Y. (1998) Convolutional networks for images, speech, and time series [M/OL]. In The Handbook of Brain Theory and Neural Networks. MIT Press.

Liu, Y., Liu, B., Wang, X. F., \& Guan, C. H. (2015). Problems and Countermeasures for Chemical Weed Control in China. Pesticide, 44(7), 289-305.

Lv, W., Dong, L., Sun, Y. H., \& Li, Y. (2018). Discussion on Weed Control Methods at Home and Abroad. Zhongguo Nongxue Tongbao, 34(11), 34-39.

Qiao, Y. L., He, D. J., Zhao, C. Y., \& Tang, J. L. (2013). Identification of Weeds in Corn Field Based on Multispectral Image and SVM. Journal of Agricultural Mechanization Research, (8), 88-91.

Shabbir, A., Bajwa, A. A., Dhileepan, K., Zalucki, M., \& Adkins, S. (2018). Integrated use of biological approaches provides effective control of parthenium weed. Archives of Agronomy and Soil Science, 64(13), 1861-1878. do i: $10.1080 / 03650340.2018 .1464150$

Su, Y. 1. (2017). Weed Control Techniques in Corn Fields. Agriculture Engineering Technology, 37(26), 25-25.

Szegedy, C., Liu, W., Jia, Y., Sermanet, P., Reed, S., Anguelov, D., ... Rabinovich, A. (2014). Going deeper with convolutions. 
Vinod, N., Geoffrey, E., \& Hinton. (2010). Rectified Linear Units Improve Restricted Boltzmann Machines. In ICML'10 Proceedings of the 27th International Conference on International Conference on Machine Learning (pp. 807-814). Academic Press.

Wang, C., \& Li, Z. W. (2016). Identification of weeds using support vector machine model with fusion height and monocular image features. Nongye Gongcheng Xuebao (Beijing), 32(15), 165-174.

Wen, Z., Ying, Y. I., \& Hou, L. (2007). Influences of different cultivation patterns under ridge plowing on maize yield effect in western dryable area of liaoning province. Maize Science, 15(5), 96-99, 103.

Xie, S. P., Zheng, X. Y., Chen, Y., Xie, L. Z., Liu, J., Zhang, Y. D., \& Hu, Y. N. et al. (2018). Artifact Removal using Improved GoogLeNet for Sparse-view CT Reconstruction. Scientific Reports, 8(1), 67-72. doi:10.1038/ s41598-018-25153-w PMID:29712978

Xie, X. B., Xiao, Y. X., Shen, Y. F., Yang, Z. B., Zhao, G. A., Meng, J. Z., ... Hu, C. L. (2018). Effects of artificial weeding on barley growth and yield. Yunnan agricultural science and technology, (S1), 130-132.

Xu, Z. P. (2011). Main types of common weeds in corn field and their prevention and control. Jilin Agriculture: Academic Edition, 2011(3), 114-114.

Yan, B. Z. (2018). Application of Machine Vision Technology in Weed Identification of Corn Seedlings. Agricultural Mechanization Research, 40(3), 214-223.

Zhai, W. X., Zhao, X. M., Wei, B., \& Wang, G. D. (2019). Recognition of liver cirrhosis by deep learning model GoolgeNet-PNN. Computer Engineering and Applications, 55(05), 112-117.

Zhang, J. H., Kong, F. T., Wu, J. Z., Qi, Z. F., Han, S. Q., \& Cao, Y. (2018). A cotton disease identification model based on improved VGG convolutional neural network. Zhongguo Nongye Daxие Хиеbao, 23(11), 161-171.

Zhao, T. \& Liu, T. Y. (2019). Research of Field Weeds Identification on Convolutional Neural Network. Computer simulation, 36(4), 440-444.

Zhong, Z., Jin, L., \& Xie, Z. (2015). High Performance Offline Handwritten Chinese Character Recognition Using GoogLeNet and Directional Feature Maps.

Zhuo, S. R., Liang, W. L., Li, J. G., \& Kim, J. (2018). Improved VGG Model for Road Traffic Sign Recognition. CMC-Computers Materials \& Continua, 57(1), 11-24. doi:10.32604/cmc.2018.02617

LiFang Fu (1974 ), Associate Professor, Research field: Intelligent Agriculture, Agricultural Big Data Science.

Xingchen Lv received the BEng from Chengdu University of Information Technology in 2016. She is currently working toward a master's degree in the Industry Engineering, the Northeast Agricultural University. Her research interests include pattern recognition, computer vision, and machine learning. 